\title{
The Effects Of Surface Hydroxyl Groups In Polyethylene-Silica Nanocomposites
}

\author{
M. Praeger, I. L. Hosier, A. S. Vaughan, S. G. Swingler \\ Tony Davies High Voltage Laboratory \\ University of Southampton \\ Southampton, U. K. \\ mattp@soton.ac.uk
}

\begin{abstract}
Logically, the surface chemistry of filler particles must be a key factor that governs how they interact with a polymer matrix, determining for example, how strongly the particles are bound into the matrix and how easy or difficult it is to achieve a homogenous dispersion of filler particles. This second point is surely one of the most basic challenges when producing a nanocomposite (poor dispersion is frequently stated as the cause of undesirable results). Many attempts have been made to modify the surface chemistry of filler particles through surface functionalization. Typically, this is achieved by chemically attaching polymer chains to the surface of the filler particles. In this paper we try a more direct approach; the surface chemistry of silica nanoparticles is modified by processing them at high temperature. This procedure removes hydroxyl groups from the surface of the filler particles, leaving siloxane groups which are stable at room temperature. Polyethylene composites were produced using both "as delivered" and high temperature processed nanosilica. After heat treatment the particles become hydrophobic which reduces the propensity for water uptake in the resulting nanocomposite and significantly modifies the dielectric response of the material.
\end{abstract}

Keywords_polyethylene; nano; silica; hydroxyl; dielectric;

\section{INTRODUCTION}

Even in the wake of current environmental concerns there is an ever increasing demand for electrical power (which is often considered "clean" at the point of use). The anticipated "new demand" (for example, due to the growing prevalence of electric vehicles) and the growth in remote power generation (such as off-shore wind) means that it is necessary to transmit larger quantities of electrical energy over longer distances than ever before. Developments in insulation materials (particularly for HVDC) potentially offer efficiency savings which will make long distance interconnects and international super-grids an attractive proposition [1]. When seeking to produce a material with enhanced properties it is natural to explore the possibility of composite materials. This has proved to be very successful for mechanical and structural materials especially where micro or nanometric domains of inorganic materials are entrained within a polymer matrix [2]. Nanodielectrics offer real potential for development of "new materials" with desirable electrical properties [3-4]; however, to date the field has been plagued with variability [5] and has failed to demonstrate repeatable improvement of electrical properties.
Historically, silica has been a popular choice as a nanocomposite filler material. As a bulk material silica has a number of favourable properties: it is non-toxic, it has a relatively high dielectric strength and electrical resistivity, and it is mechanically strong but can be flexible when formed into a fibre. Furthermore, silica is widely available in nanometric sizes, and can be produced at low cost by flame hydrolysis. Unfortunately silica is also hygroscopic and water soluble; water contamination is therefore very likely to modify the electrical properties of the resulting nanodielectrics.

Moisture contamination can be present in several forms within a sample of silica nanopowder [6]. These forms can be distinguished by the temperature needed to remove the impurity. Free liquid water is evaporated off at $100^{\circ} \mathrm{C}$. Once physically adsorbed onto the surface of the silica additional activation energy is required for water desorption; temperatures between $150{ }^{\circ} \mathrm{C}$ and $200^{\circ} \mathrm{C}$ are needed to remove this moisture. Above this temperature the moisture contamination takes the form of surface hydroxyl groups. Vicinal silanols are removed by temperatures of $450{ }^{\circ} \mathrm{C}$ to $500{ }^{\circ} \mathrm{C}$ whilst germinal and single groups require still higher temperatures. When they reach a sufficiently high temperature these hydroxyl groups condense to form strained siloxane groups; these are readily rehydroxylated if exposed to water, however, if the silica is further heated to temperatures between $1000{ }^{\circ} \mathrm{C}$ and $1100{ }^{\circ} \mathrm{C}$ the strained siloxane groups will reconfigure and become stable. Once the silica surface is terminated in this way it requires considerable energy to rehydroxylate; the silica therefore becomes stable in its dehydroxylated state.

Silica nanoparticles are commonly produced by flame hydrolysis (fumed silica). In this process, the precursor gas, silicon tetrachloride, is burned in a hydrogen-oxygen flame in order to produce nanoscale spherical silica particles. Note that in this process the silica is exposed to high temperatures, hydrogen and oxygen. As a result the nanoparticles are typically in a hydroxylated state.

\section{METHOD}

\section{A. Nanoparticle preparation}

In this study silica nanopowder of type Aldrich 637238 was used; this will be referred to as the "as delivered" (AD) silica filler. Some of the same material was placed in a furnace and 
held at $1050{ }^{\circ} \mathrm{C}$ under dry nitrogen for 10 hours; this will be referred to as the "high-temperature processed" (HTP) filler. Both the AD and HTP fillers were stored under ambient lab conditions for several months prior to testing and undoubtedly contain some surface adsorbed moisture.

\section{B. Nanocomposite sample preparation}

The polymer matrix used in this study is a 20:80 wt $\%$ blend of HDPE (BP Rigidex HD5813EA) and LDPE (ExxonMobil LD100BW) respectively. A master batch of this blend was produced in a mechanical melt mixer. For each sample, $5 \mathrm{~g}$ of PE blend was dissolved in $50 \mathrm{ml}$ of hot xylene. $0.25 \mathrm{~g}$ of each nanosilica powder was dispersed in $12 \mathrm{ml}$ of isopropyl alcohol (IPA) by probe sonicating for 10 minutes. The xylene-polymer mixture was taken off the heat and the nanoparticle solution was added whilst vigorously mixing. The IPA rapidly evaporates transferring the nanoparticles into the xylene. As the xylene and polymer cools it quickly becomes more viscous which prevents the nanoparticles from re-agglomerating or settling out. This resulted in nanocomposite samples with a filler loading of nominally $5 \%$.

The sample material was dried in a vacuum oven to remove residual solvent and was then pressed into flat discs approximately $100 \mu \mathrm{m}$ in thickness. Gold electrodes were sputter coated onto both sides of each sample to ensure good electrical contact during the dielectric measurements.

Upon visual inspection, both types of nanofiller appear to be very well dispersed within the polymer. There are no large agglomerates visible by eye and despite the $5 \%$ filler loading both samples appeared to be completely transparent during hot pressing (prior to crystallisation).

\section{Nanocomposite sample conditioning}

Altering the number density of hydroxyl groups on the surface of a silica nanoparticle is expected to strongly influence its interaction with water (changing the particle from hydrophilic to hydrophobic). It is therefore interesting to investigate how samples produced with the AD and HTP fillers differ in their interaction with water. To this end, samples were conditioned either by soaking in water or by drying in a vacuum oven for one week. The properties of the conditioned samples can then be compared with those of samples that have simply been kept in the ambient laboratory environment.

\section{Fourier transform infrared analysis}

Fourier transform infrared (FTIR) spectroscopy was used in order to quantify the observed differences between the nanosilica types. FTIR measurements were made using a Perkin Elmer Spectrum GX, with an AutoIMAGE microscope attachment, operated in reflection mode. Samples for FTIR were made by sonicating both types of nanosilica in dichloromethane (DCM); these solutions were then dripped onto gold coated microscope slides.

\section{E. Thermogravimetric Analysis (TGA)}

A Perkin Elmer Pyris 1 TGA was used to perform thermogravimetric analysis. The temperature was ramped at a rate of $10^{\circ} \mathrm{C} / \mathrm{min}$ up to a maximum of $650^{\circ} \mathrm{C}$ in an atmosphere of dry air. During this process the polymeric fraction of the sample is combusted whilst the inorganic filler is left as a residual mass.

\section{F. Dielectric spectroscopy}

The dielectric response of the nanocomposite samples was measured with a Solartron frequency response analyser (model 1260), associated dielectric interface (model 1296) and sample holder (model 12962). The electrodes are fitted with a guard ring and have a circular measuring region that is $30 \mathrm{~mm}$ in diameter.

\section{RESULTS}

\section{A. Observations on the effectiveness of thermal treatment}

The first undertaking of this work is to establish whether or not high temperature processing has modified the properties of the nanosilica particles. Indeed, qualitative differences were already observed when each of the nanoparticle types was dispersed into IPA. Upon stirring the AD nanosilica immediately formed a visually homogenous dispersion. In contrast, after stirring the HTP nanosilica was visibly agglomerated into millimetre scale flock-like particles. This observation is consistent with the theory that the HTP nanosilica, having been dehydroxylated, is more hydrophobic. After sonication the solutions of both nanoparticle types had a uniform opaque white appearance.

\section{B. Fourier transform infrared}

Fig. 1 shows the resulting FTIR spectra, which display clear differences between the two filler types. One of the most obvious features is a very broad absorption between 3000 and $3700 \mathrm{~cm}^{-1}$ which is far more intense for the AD fillers than for the HTP ones. In part, this band can be attributed to stretching modes of hydrogen-bonded $\mathrm{OH}$ groups [7-8]. The IR response of these groups is highly dependent upon local geometric restrictions and, for nanofillers, the FTIR microscope integrates over a diverse range of these local conditions. This goes some way to explaining the extreme width of this absorption band; however, it is likely that some liquid (surface adsorbed) water is also present.

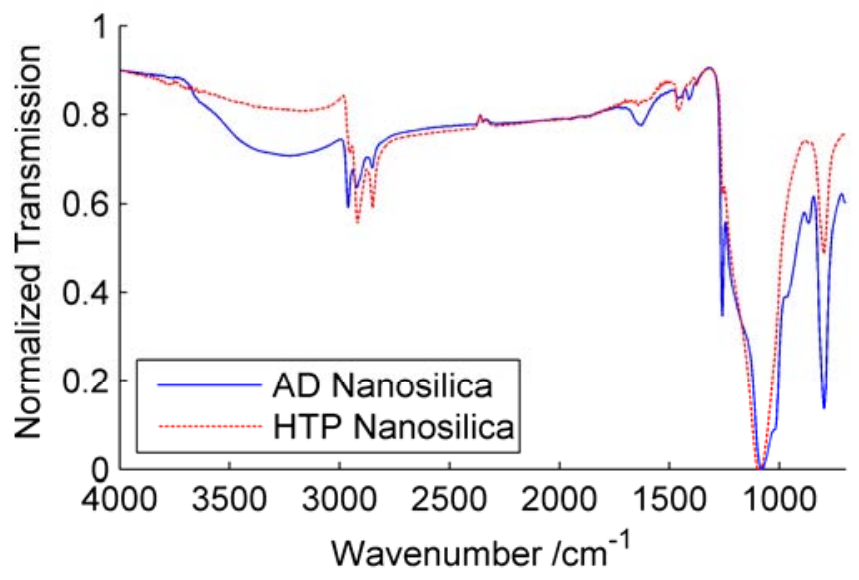

Fig. 1. FTIR spectra of HTP and AD nanosilica, measured using a microscope attachment in reflection mode. 
Further evidence for the presence of water is given by the small absorption peak at $1635 \mathrm{~cm}^{-1}$ and by the broadening of the main absorption peak centred near $1080 \mathrm{~cm}^{-1}$. The sharp peak near $1260 \mathrm{~cm}^{-1}$ may indicate a slight residue of DCM.

Whilst using the microscope to take FTIR measurements it was observed that the HTP nanosilica tended to form larger clumps with very little particulate material between. The AD nanosilica particles, on the other hand, appeared to be spread more evenly over the whole of the droplet sample region (see Fig. 2). Again, this is consistent with the HTP fillers being hydrophobic whilst the AD filler particles are hydrophilic.
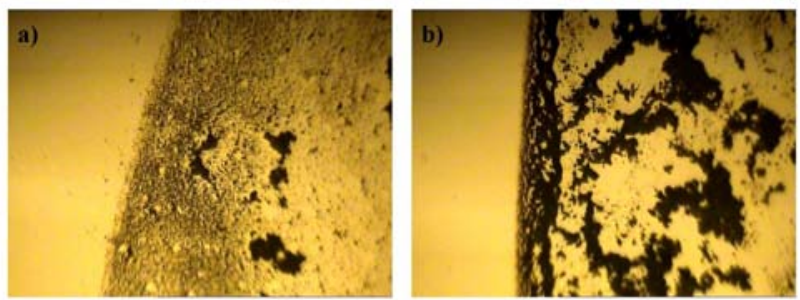

Fig. 2. Microscope images of the filler residue after dripping nanosilicaDCM solution onto a gold coated microscope slide. a) the AD particles seem to be spread more uniformly. b) The HTP particles have a very strong tendency to clump together and form large dense agglomerates.

\section{Thermogravimetric Analysis (TGA)}

In some sample preparation methods (for example where precipitation in non-solvent is used as a means of recovering the polymer) there is a chance that a fraction of the filler content will be lost during production. The method used here does not have this drawback and the TGA data (see Fig. 3) indicates that the filler loading is very close to the $5 \%$ w.t. target.

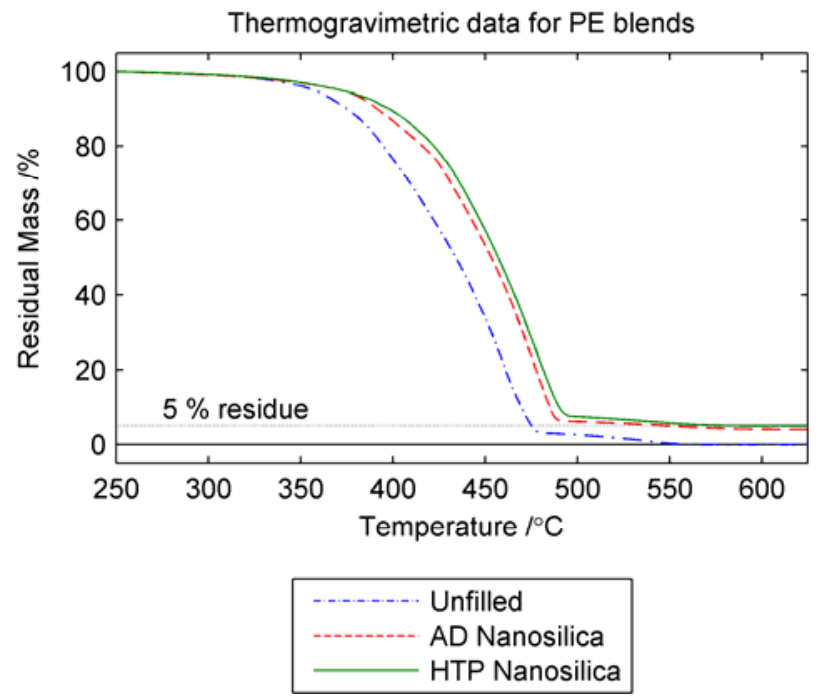

Fig. 3. TGA profiles for the unfilled PE blend (dash dot curve) and for the two nansilica composites, AD and HTP dashed and solid curves respectively.

Both filler types (AD and HTP) have a reinforcing effect which allows their composites to survive to slightly higher temperatures than the unfilled polymer. When comparing the $\mathrm{AD}$ and HTP nanosilica composites it is evident that the AD filler loses mass more rapidly between 400 and $450{ }^{\circ} \mathrm{C}$. This is consistent with removal of bound water through condensation of silanol groups from the $\mathrm{AD}$ nanosilica. The final residual mass for the HTP sample is $5 \%$ w.t. whilst for the AD sample it is $4.1 \%$ w.t. The lower filler loading for the AD sample can be explained if water content makes up part of the initially measured filler mass.

\section{Change of sample mass upon conditioning}

The simplest method to quantify the amount of water uptake or egress during conditioning is by monitoring the sample mass. These data are expressed as a percentage change relative to the initial sample mass in table 1 .

TABLE I. CHANGE IN SAMPLE MASS DUE TO CONDITIONING

\begin{tabular}{|c|c|c|}
\hline \multirow{2}{*}{$\begin{array}{c}\text { Nanosilica } \\
\text { type }\end{array}$} & \multicolumn{2}{|c|}{ Average mass change for each conditioning type } \\
\hline & Vacuum drying & Water soaking \\
\hline $\mathrm{AD}$ & $-0.98 \% \pm 0.01$ & $+0.49 \% \pm 0.10$ \\
\hline HTP & $-0.88 \% \pm 0.27$ & $+0.02 \% \pm 0.05$ \\
\hline
\end{tabular}

Upon vacuum drying for one week both the AD and HTP nanocomposites lose approximately $1 \%$ of their mass. Although much of this mass change is thought to be due to a reduction of water content there may also be some further removal of residual solvent. The slightly larger mass reduction seen for the $\mathrm{AD}$ samples is probably indicative of a higher initial water content. When immersed in water for one week the $\mathrm{AD}$ nanocomposite increases its mass by approximately $0.5 \%$ whilst the mass of the HTP sample remains almost unchanged. The total range of mass change is higher for the AD samples indicating a higher propensity to change their moisture content than the HTP samples. In comparison the HTP nanosilica tends to absorb much less water upon immersion; this may be a result of the hydrophobicity that was commented on previously.

\section{E. Dielectric properties as a function of conditioning}

Dielectric spectra were initially recorded for all samples whilst they were in equilibrium with the ambient laboratory conditions (see Fig. 4). The AD nanocomposite exhibits a large dielectric loss peak centred at approximately $200 \mathrm{~Hz}$; there may also be a shoulder at higher frequencies. For the HTP nanocomposite the dielectric loss is low $\left(\tan (\delta)<1 \times 10^{-2}\right)$ across the entire measured range. Interestingly, the opposite effect has been reported for $\mathrm{BaTiO}_{3}$ cyanate ester composites [9], where the dried filler displayed a higher dielectric loss.

The dielectric spectra were re-measured after conditioning for one week either by vacuum drying or by soaking in water (see Fig. 5). For the AD samples, immersion in water shifts the loss peak to higher frequencies whilst drying them shifts the loss to very low frequencies.

The behaviour of the dried HTP samples is similar to that of the ambient HTP ones although with even lower dielectric loss. After immersion in water for one week the HTP sample has developed a loss peak centred just below $10 \mathrm{~Hz}$, with a shoulder extending to higher frequencies. The amplitude of the main loss peak remains lower than those observed for the $\mathrm{AD}$ fillers. 


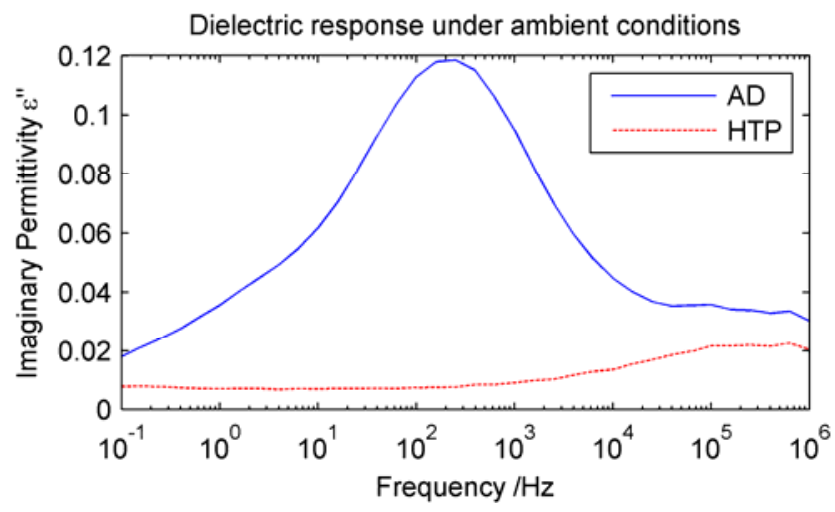

Fig. 4. Imaginary component of permittivity $\varepsilon$ " for the AD (solid) and HTP (dotted) nanocomposite samples (in equilibrium with ambient conditions).

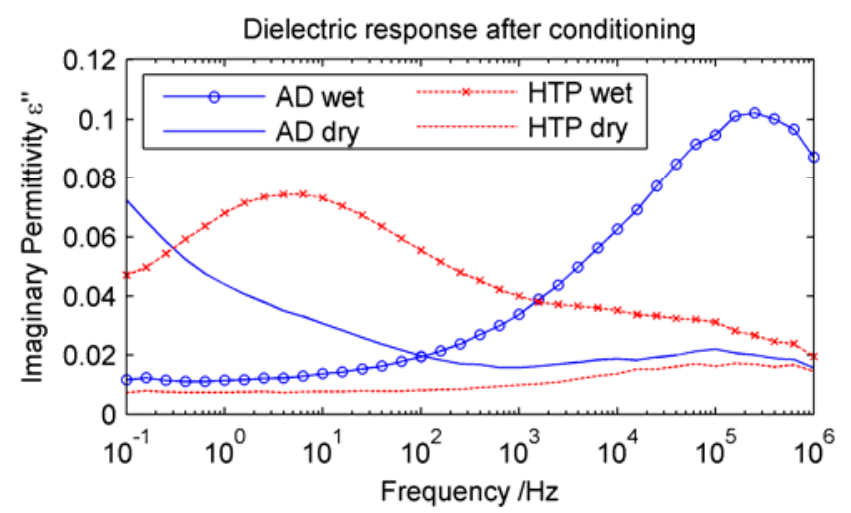

Fig. 5. Imaginary component of permittivity $\varepsilon$ " for the AD (solid) and HTP (dotted) nanocomposite samples after vacuum drying (no markers) and after soaking in water (markers).

\section{CONCLUSION}

High temperature processing has been shown to make a measurable and enduring difference to the surface chemistry of silica nanoparticles. Observations confirmed that the particles changed from hydrophilic to hydrophobic in nature whilst FTIR indicated reduced water content and fewer hydroxyl groups after processing. The TGA data reveal that an appreciable fraction of the mass of the $\mathrm{AD}$ nanosilica consists of bound water and surface silanols. Temperatures exceeding $400{ }^{\circ} \mathrm{C}$ are required to remove this moisture; this means that it is normally not possible to remove this from the nanocomposite without damaging the polymer matrix.

The adopted method of incorporating nanofiller into the polymer blend was very successful. Visually, the dispersion of filler particles was extremely uniform with no evidence of large agglomerates. It is proposed that the hydrophobic HTP filler interacts favourably with the non-polar PE in order to further aid dispersion but further testing (possibly TEM) is needed to confirm this.

The sample mass change upon conditioning shows that the $\mathrm{AD}$ nanosilica has a higher total capacity for water storage. In contrast the hydrophobic nature of the HTP fillers produces nanocomposites with a far lower propensity to absorb water.

The dielectric response is extremely sensitive to the water content of the samples; loss peaks tend to shift towards higher frequencies as water content is increased. After the same conditioning the hydrophobic HTP fillers always demonstrated lower loss than the AD equivalent. Even after being soaked in water for a week the HTP sample outperformed the ambient $\mathrm{AD}$ one over most of the measured frequency range.

The main loss peak and its associated high frequency shoulder may represent two distinct relaxation processes; one corresponding to silanol groups whilst the other is related to physically adsorbed water which forms a water shell at the silica surface as discussed in [10].

This work shows that eliminating water from nanocomposites is vital to ensure good electrical properties. Water must be eliminated from the filler particles prior to making the nanocomposite or it will be locked in place. If nanocomposites can be engineered to be intrinsically water repellent then this could help to maintain excellent electrical properties even whilst operating in challenging environments for extended periods. High temperature processing has been shown to be an effective means of modifying the surface chemistry of silica nanopowders and offers a viable alternative to surface functionalisation.

\section{ACKNOWLEDGMENT}

The authors gratefully acknowledge the RCUK's Energy Programme for the financial support of this work through the Top \& Tail Transformation programme grant, EP/I031707/1 (http://www.topandtail.org.uk/).

\section{REFERENCES}

[1] T. J. Hammons, V. F. Lescale, K. Uecker, M. Haeusler, D. Retzmann, K. Staschus, et al., "State of the Art in Ultrahigh-Voltage Transmission," Proc. IEEE, vol. 100, pp. 360-390, Feb 2012.

[2] S. C. Tjong, "Structural and mechanical properties of polymer nanocomposites," Mater. Sci. Eng., R, vol. 53, pp. 73-197, Aug 2006.

[3] T. J. Lewis, "Nanometric Dielectrics," IEEE Trans. Dielectr. Insul., vol. 1, pp. 812-825, Oct 1994.

[4] M. F. Frechette, A. Vijh, M. L. Trudeau, D. Fabiani, L. Utracki, S. Gubanski, et al., "Nanodielectrics: A panacea for solving all electrical insulation problems?," in Solid Dielectrics (ICSD), 2010 10th IEEE International Conference on, 2010, pp. 1-29.

[5] I. L. Hosier, M. Praeger, A. F. Holt, A. S. Vaughan, and S. G. Swingler, "Effect of water absorption on dielectric properties of nanosilica/polyethylene composites," 2014 IEEE Conference on Electrical Insulation and Dielectric Phenomena (CEIDP), pp. 651-654, 20142014.

[6] B. Horacio, "Colloid Chemistry of Silica," in Colloidal Silica, ed: CRC Press, 2005, pp. 9-35.

[7] R. S. McDonald, "Surface Functionality of Amorphous Silica by Infrared Spectroscopy," J. Phys. Chem., vol. 62, pp. 1168-1178, 1958.

[8] P. Innocenzi, "Infrared spectroscopy of sol-gel derived silica-based films: a spectra-microstructure overview," J. Non-Cryst. Solids, vol. 316, pp. 309-319, Feb 2003.

[9] F. Chao, N. Bowler, X. L. Tan, G. Z. Liang, and M. R. Kessler, "Influence of adsorbed moisture on the properties of cyanate ester/BaTiO(3) composites," Composites Part a-Applied Science and Manufacturing, vol. 40, pp. 1266-1271, Aug 2009.

[10] J. G. Meier, J. Fritzsche, L. Guy, Y. Bomal, and M. Klueppel, "Relaxation Dynamics of Hydration Water at Activated Silica Interfaces in High-Performance Elastomer Composites," Macromolecules, vol. 42, pp. 2127-2134, Mar 242009. 